\title{
Structurally Anisotropic Janus Particles with Tunable Amphiphilicity via Polymerization of Dynamic Complex Emulsions
}

\author{
Bradley D. Frank, Markus Antonietti, and Lukas Zeininger*
}

Cite This: Macromolecules 2021, 54, 981-987

Read Online

ABSTRACT: A facile one-step approach for the synthesis of physically and chemically anisotropic polymer particles with tunable size, shape, composition, wettability, and functionality is reported. Specifically, dynamically reconfigurable oil-in-water Janus emulsions containing photocurable hydrocarbon or fluorocarbon acrylate monomers as one of the droplet phases are used as structural templates to polymerize them into precision Janus particles with highly uniform anomalous morphologies including (hemi-) spheres, lenses, and bowls. During polymerization, each interface is exposed to a different chemical environment, yielding particles with an intrinsic Janus character that can be amplified via side-selective postfunctionalization. The fabrication method allows to start with various common emulsification techniques, thus generating particles in the range of $200 \mathrm{~nm}-150 \mu \mathrm{m}$, also at a technical scale. The anisotropic shape combined with the asymmetric wettability profile of the produced particles promotes their directed self-assembly into colloidal clusters as well as their directional alignment at fluid interfaces. We foresee the application of such Janus particles in technical emulsions or oil recovery, for the manufacturing of programmed self-assembled architectures, and for the engineering of microstructured interfaces.

\section{INTRODUCTION}

Polymer particles with two distinct faces of differing chemistries, so-called Janus particles, represent a unique collection of functional colloidal materials featuring anisotropic wettability, chemistry, and optical, electric, and magnetic properties. ${ }^{1,2}$ Due to their amphiphilicity and thus directionality within a single hybrid particle, Janus particles have attracted considerable attention in a diverse set of applications, including as powerful solid surfactants to mediate stability in multiphasic fluid mixtures and influence the packing dynamics at the interfaces, ${ }^{3-7}$ as motile particles with directional propulsion profiles, ${ }^{8,9}$ colloidal building blocks for selfassembled structure formation, ${ }^{10-12}$ for imaging, ${ }^{13,14}$ or as transducers in sensing applications..$^{15-17}$ Because the properties and functions of Janus particles are closely related to their geometry and composition, a variety of fabrication methods have been developed to meet specific application-oriented requirements. $^{18}$ Most common techniques rely on either breaking the symmetry of existing particles or identifying the pathways to directly synthesize or self-assemble smaller components into anisotropic structures.

Most nano- and microscale Janus particles are produced via selective-growth techniques, where particles are restrained on a two-dimensional interface and subsequent side-selective postmodification generates asymmetric particles. ${ }^{19}$ While this method is straightforward, and well-defined Janus particles can be generated, compositional flexibility and upscaling of this approach is limited to the milligram to gram scale. Alternatively, particles have been produced via self-assembly of specifically designed block copolymers. Cross-linking of selfassembled polymer bundles facilitates the generation of nanoscale soft-matter Janus colloids in solution. ${ }^{20-22}$ However, methods of this nature have difficulty addressing larger microscale particles and often involve tedious syntheses and the diversity of accessible particle shapes is still rather limited.

Photoinduced radical polymerization within the confined space of emulsion droplets has evolved as a powerful alternative for the production of anisotropic particles and supraparticles with remarkable control of shape and functionality. ${ }^{23-27}$ Emulsion and dispersion polymerization are the classical methods for the production of polymers and are industrially applied on the largest scale by utilizing the compartmentalization of emulsion droplets for control of polymer molecular weight, viscosity, and heat flux..$^{28-31}$

Besides single-phase emulsion droplets, the use of complex emulsion droplets such as multiple, multilayered, and Janus emulsions gives rise to a large structural diversity, which can be polymerized to produce anisotropic particles. ${ }^{32-35}$ As a result, microfluidic techniques, in particular, have evolved as a powerful tool for the generation of monodisperse micrometer-sized anisotropic particles, as these approaches allow for the creative design of numerous geometrically complex and intricate objects. $^{36,37}$ As a drawback, microfluidic approaches typically require multiple fabrication steps, are limited to the higher micrometer size range, and depend on hard or soft lithographic techniques for the fabrication of highly sophisti-

Received: September 16, 2020

Revised: November 24, 2020

Published: December 7, 2020 


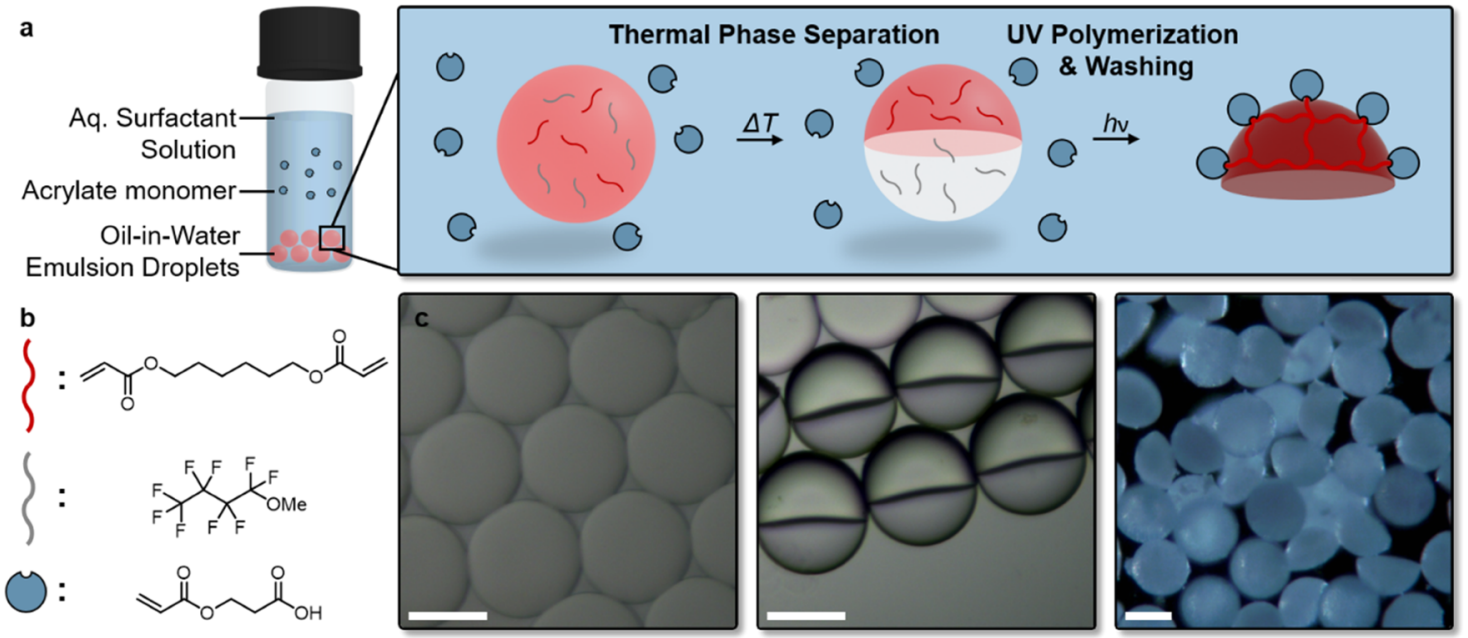

Figure 1. One-step fabrication of chemically and physically anisotropic Janus particles. (a) Conceptual sketch of the fabrication procedure. (b) Chemical structures of acrylate monomers and solvents used in this method. (c) Side-view micrographs of the three different stages during the fabrication procedure. Scale bar: $100 \mu \mathrm{m}$.

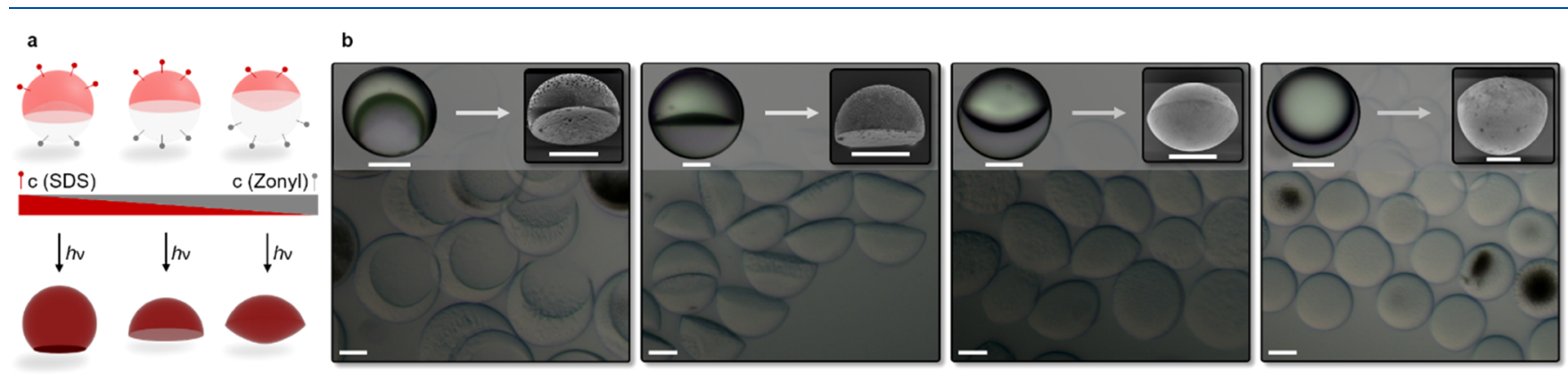

Figure 2. Production of anisotropic particles via phase-selective emulsion polymerization. (a) Dynamic configurability of Janus emulsion morphology via surfactants and subsequent conversion into precision anisotropic particles. (b) Side-view optical micrographs of produced anisotropic particles with insets showing side-view micrographs of Janus droplets before polymerization, and scanning electron micrograph of the resulting particles. Scale bars: $50 \mu \mathrm{m}$.

cated microfluidic channels, and upscaling is tied to the number of microfluidic channels run in parallel. Consequently, practical applications of Janus colloids are still hindered by missing accessibility and structural flexibility.

Herein, we describe a novel, one-step, bulk approach to generate chemically and physically anisotropic particles based on a selective polymerization of one phase inside oil-in-water Janus emulsions. The internal morphology of complex emulsions comprised of acrylate monomers as one of the droplet phases can be controlled by balancing the individual interfacial tensions using surfactants. Dynamic control of these droplet templates allows the creation of intricate particle shapes such as (hemi-) spheres, lenses, and bowls in high uniformity across a sample, with size ranges and distributions solely controlled by the emulsification method (Figure 1).

\section{RESULTS AND DISCUSSION}

To synthesize a broad variety of Janus polymer particles via phase-selective emulsion polymerization, we first prepared dynamically reconfigurable Janus emulsions with photocurable acrylates as one of the constituent liquid phases. To this end, we selected the hydrocarbon monomer 1,6-hexanediol diacrylate (HDDA) and the fluorocarbon oil methoxyperfluorobutane (FC) as our constituent droplet phases. Mixtures of these two liquids become homogeneous with gentle heating $\left(T_{\mathrm{C}} \sim 35^{\circ} \mathrm{C}\right)$, but de-mix at room temperature (Figure $2 \mathrm{a}$ ).
Using an established thermal phase separation approach, ${ }^{38}$ Janus emulsions were readily obtained by emulsifying a 1:1 volume ratio of the two oils above their $T_{\mathrm{C}}$ in an aqueous solution containing a mixture of surfactants. Depending on the surfactant composition in the continuous phase, the internal morphologies of these complex emulsions could be dynamically altered between encapsulated (HDDA/FC/W and FC/ HDDA/W), and Janus emulsion geometries (Figure S1). This is done by varying the interfacial tensions at the external hydrocarbon and fluorocarbon interfaces using different ratios of hydrocarbon and fluorocarbon surfactants, namely, sodium dodecyl sulfate (SDS) and Zonyl FS-300, respectively (Figure 2a).

Janus droplets in different morphologies were then polymerized into their corresponding anisotropic particle replicas. To this end, we added a radical photoinitiator (2hydroxy-2-methylpropiophenone, Darocur 1173) and placed the as-prepared Janus emulsions under a UV lamp. We observed that the photoinitiated polymerization of the monomer phase proceeded from the external interface of the droplets inward, which can be attributed to the partial solubility of the radical initiator within the continuous phase (Figure S2). As a result, the shape and size of the monomer droplet phase were essentially retained and locked upon polymerization (Figure $2 \mathrm{~b}$ ). Utilizing the same thermal phase separation approach, particles of different compositions were 
obtained employing 1,4-butanediol diacrylate (BDDA) or lauryl acrylate (LA) as alternative hydrocarbon monomers, or perfluorodecyl acrylate (PFDA), with a hydrocarbon solvent to yield the fluorocarbon particle analogues (Table 1).

Table 1. Solvent and Monomer Combinations Used for the Production of Janus Particles in This Study

\begin{tabular}{cclcc} 
monomer phase & monomer density & oil phase & oil density & $T_{\mathrm{C}}\left({ }^{\circ} \mathrm{C}\right)$ \\
HDDA & 1.01 & $\mathrm{MeOC}_{4} \mathrm{~F}_{9}$ & 1.52 & 35 \\
BDDA & 1.05 & $\mathrm{MeOC}_{4} \mathrm{~F}_{9}$ & 1.52 & 24 \\
LA & 0.88 & $\mathrm{MeOC}_{4} \mathrm{~F}_{9}$ & 1.52 & 33 \\
PFDA & 1.63 & octane & 0.70 & 24 \\
PFDA & 1.63 & decane & 0.73 & 49 \\
\hline
\end{tabular}

In principle, this approach is applicable to any conventional emulsification technique that allows variation of the resulting particle sizes and size distributions over a wide range. As such, we obtained large quantities of microsized Janus particles $(d \sim$ $100 \mu \mathrm{m})$ starting from macroemulsions prepared using largescale, batch techniques such as hand-shaking or vortex mixing. Applying more precise microfluidic methods yielded microsized Janus particle samples with low size distributions $(<5 \%)$. In turn, when we applied high-energy emulsification techniques such as homogenization or ultrasonication (Table S1), we obtained small Janus particles in size ranges of $d=15-$ $27 \mu \mathrm{m}$ or $d=294-967 \mathrm{~nm}$, respectively (Figure 3 ).

All polymer particles synthesized by this method display an intrinsic Janus character as each interface is exposed to a different chemical environment during polymerization. To amplify this Janus character, we added water-soluble carboxyor poly(ethylene glycol) (PEG)-functionalized acrylate monomers to the aqueous continuous phase (Table 2). On polymerization, these monomers copolymerized onto the external particle-water interface selectively, whereas the internal interface was shielded by the fluorocarbon oil phase. Scanning electron microscopy (SEM) images of amphiphilic particles produced using poly(ethylene glycol) diacrylate displayed a strong contrast in surface roughness of the inner and outer interfaces (Figure 4a). To estimate the wettability of the individual Janus particle surfaces, we produced drop-cast polymer thin films as models that were exposed to the same external phase compositions and monitored the two-dimensional (2D) static contact angle of water on those functionalized polymer substrates. On those substrates, we measured
Table 2. 2D Static Contact Angle of Water on Various Acrylate-Functionalized Polymer Thin Films

\begin{tabular}{clc}
$\begin{array}{c}\text { polymer } \\
\text { substrate }\end{array}$ & \multicolumn{1}{c}{ surface functionality } & $\begin{array}{c}\text { 2D static contact angle of } \\
\text { water (degree) }\end{array}$ \\
\hline HDDA & none & $76 \pm 1$ \\
HDDA & PEG methacrylate (360) & $49 \pm 2$ \\
HDDA & PEG methyl ether & $47 \pm 1$ \\
& methacrylate (475) & $46 \pm 1$ \\
HDDA & PEG diacrylate (700) & $45 \pm 2$ \\
HDDA & 2-carboxy-ethyl acrylate & $120 \pm 3$ \\
PFDA & none & $77 \pm 4$ \\
PFDA & 2-carboxy-ethyl acrylate & \\
\hline
\end{tabular}

the water contact angles between $\sim 40$ and $120^{\circ}$ (Table 2 ) for different monomer combinations, which illustrates the tunable hydrophilicity of the external particle surface. To ascertain the presence of the anticipated polarity contrast on the surfaces of the particles, we next investigated their behavior at fluid interfaces. At high concentrations, all produced particles were able to stabilize both oil-in-water and water-in-oil emulsions depending on the applied volume ratios of the two phases.

At low particle concentrations $(\sim 1 \mathrm{mg} / \mathrm{L})$, we observed that the Pickering emulsion stability was dependent on the amphiphilicity of the Janus particles, with more amphiphilic particles being superior over their counterparts, in which amphiphilicity was not chemically enhanced. Notably, in addition to the amphiphilicity of the particles, their shape played a significant role in promoting the stability of emulsion droplets (Figure 4b). The latter can be attributed to the differing packing dynamics of these particles at fluid interfaces. $^{39,40}$ $\zeta$-Potential measurements (Figure 4c) on differently shaped Janus particles revealed a large difference of $\Delta \xi=25 \mathrm{mV}$ in the $\zeta$-potential of cup- vs lens-shaped amphiphilic particles in aqueous solution (Figure 4c), which further supported the shape contribution to the amphiphilicity of the particle system. An increase in carboxylate-functionalized surface area and thus a change in the Janus ratio, i.e., hydrophilic to the hydrophobic surface area of the particles, resulted in an enhanced wettability contrast of the Janus particles. The latter influenced the particles' colloidal aggregation behavior in aqueous solutions to which the pronounced difference in measured $\zeta$-potential values could be attributed.

The directional organization hypothesis was supported by the side-view micrographs of amphiphilic particles located at


Figure 3. Size of the produced anisotropic Janus particles is determined by the emulsification technique. (a) Average particle sizes of the produced Janus particles depending on the emulsification method; gray areas represent the range of sizes produced in different methods; and error bars represent the polydispersities of the respective samples. (b) SEM of nanoscale hemispherical Janus particles produced via miniemulsion polymerization; scale bar: $500 \mathrm{~nm}$. 

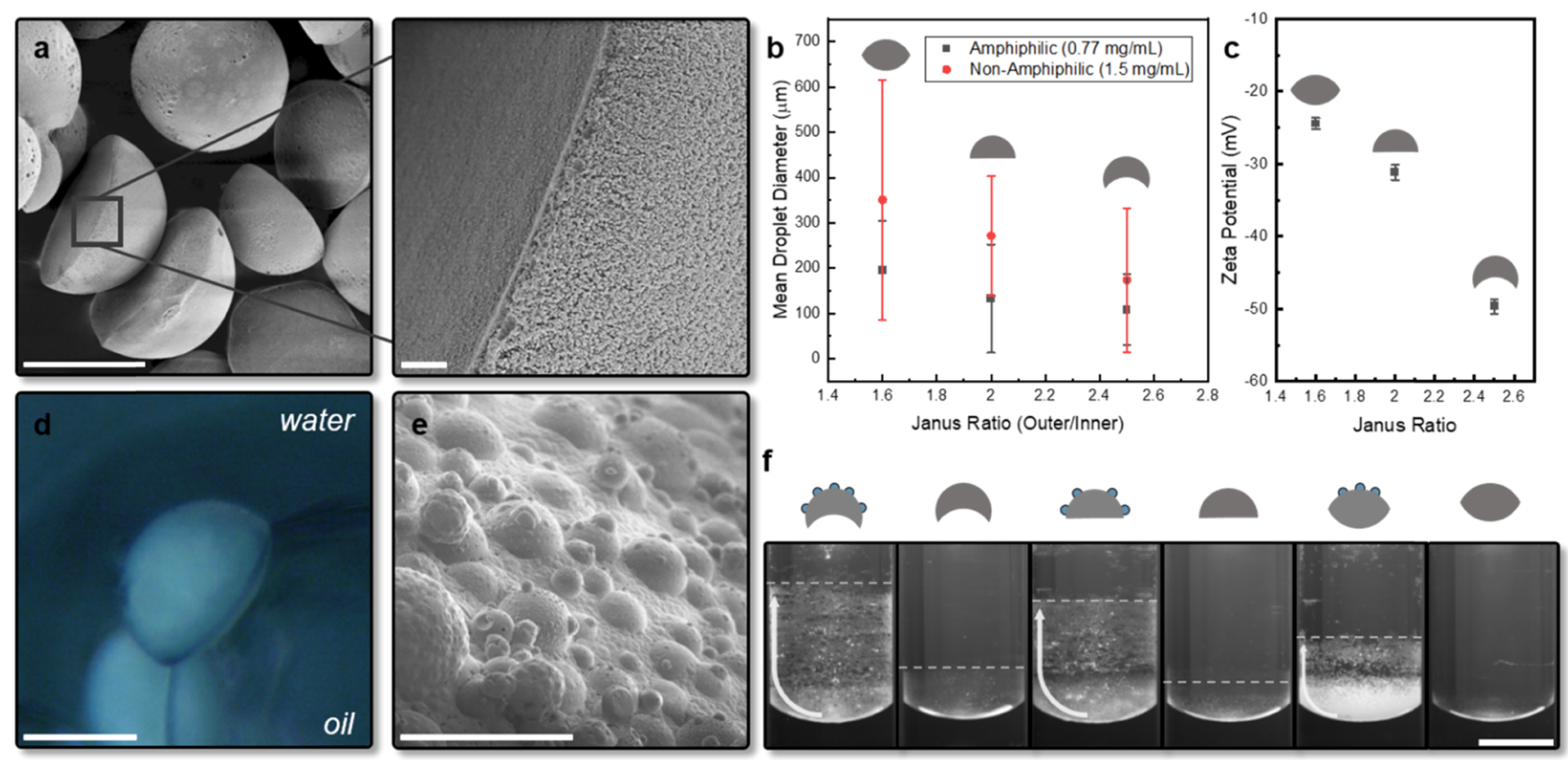

Figure 4. Tunable amphiphilicity of the produced Janus particles. (a) SEM of an amphiphilic hemispherical Janus particle (scale bar: $100 \mu \mathrm{m}$ ) showing different surface roughness for poly(ethylene glycol) diacrylate and nonfunctionalized interfaces of the particle (scale bar magnified SEM: $4 \mu \mathrm{m})$; (b) average droplet diameter of Pickering emulsions produced with amphiphilic $(0.77 \mathrm{mg} / \mathrm{mL})$ and nonamphiphillic $(1.5 \mathrm{mg} / \mathrm{mL}) \mathrm{cup}$ and hemispherical and lens-shaped HDDA particles; the emulsion sizes are plotted as a function of the particles' Janus ratio, which is the ratio of hydrophilic to hydrophobic particle surface area (see Supporting Information for details); (c) $\zeta$-potential of differently shaped amphiphilic Janus particles $(d=1.01 \pm 0.23 \mu \mathrm{m})$; (d) anisotropic wettability profile causes an directional alignment of the as-produced Janus particles at oil-water interfaces; here, inverted optical micrograph of an amphiphilic HDDA hemisphere at a water-perfluorohexane interface, scale bar: $100 \mu \mathrm{m}$; (e) polymerized PFDA droplet stabilized by hemispherical amphiphilic particles, scale bar: $100 \mu \mathrm{m}$; (f) Marangoni drag of particles with different shapes and amphiphlicities; scale bar: $1 \mathrm{~cm}$.
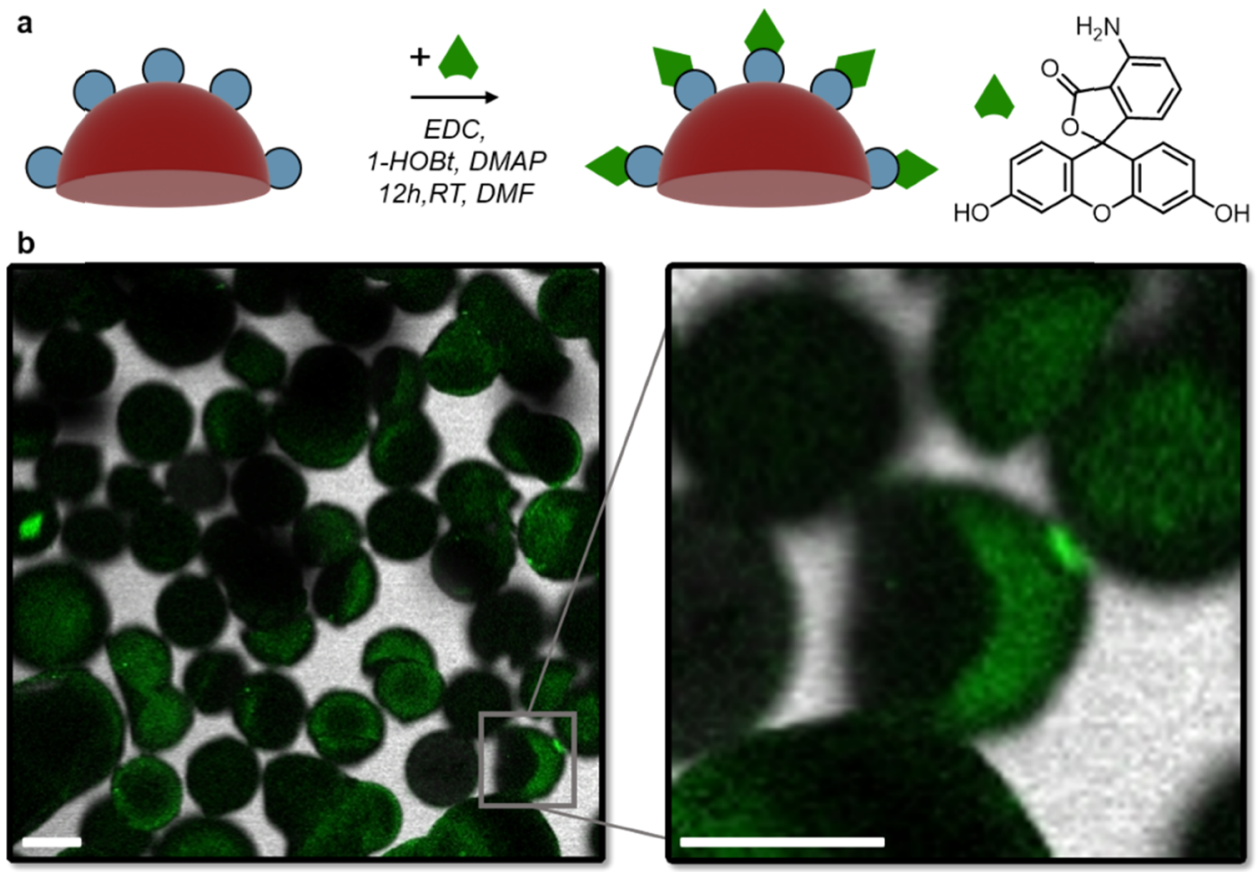

Figure 5. Side-selective postfunctionalization of amphiphilic Janus particles. (a) Reaction scheme and chemical structure of fluoresceinamine. (b) Confocal micrographs of fluoresceinamine-functionalized hemispherical Janus particles stabilized in $1 \mathrm{wt} \%$ poly(ethylene oxide), displaying the fluorescent marker to be only located at the external interfaces of the particles, scale bar: $100 \mu \mathrm{m}$.

an oil-water interface, as displayed in Figure 4d, where the differing wettability of the particles led to a directional interfacial organization at the fluid interface. The preferential alignment at the droplet interfaces was maintained also throughout a solidification process when we UV-polymerized particle-stabilized droplets composed of a fluorinated acrylate 
a



b

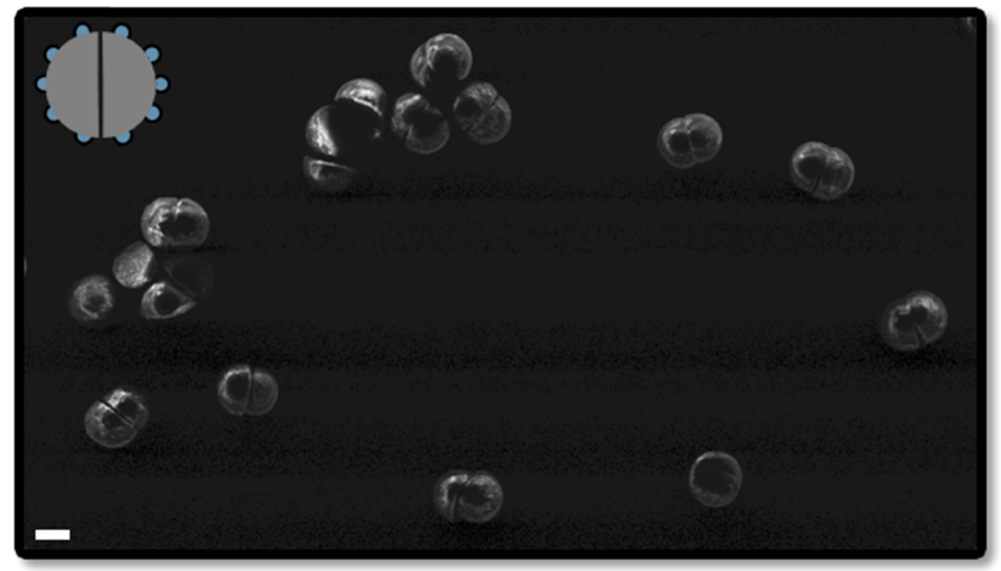

Figure 6. Colloidal self-assembly of Janus particles directed through shape and amphiphilicity. (a) Side-view micrograph of shape-directed assembly of the cup-shaped HDDA particles in toluene; scale bar: $50 \mu \mathrm{m}$. (b) Scanning electron micrograph of forced-immersion-assisted self-assembled particle dimers comprised of amphiphilic hemispherical PFDA Janus particles with water removed after deposition onto the SEM substrate; scale bar: $100 \mu \mathrm{m}$.

oil. SEM images of the surfaces of such polymer beads (Figure $4 \mathrm{e})$ revealed a largely uniform alignment of hemispherical amphiphilic particles, which served as a tool to structure the polymer particle surfaces. Moreover, the strong shape- and functionality-dependent interfacial activity of the various amphiphilic Janus particles could be demonstrated by comparing the Marangoni drag of particles up the wall of a glass vial when located at an oil-water fluid interface (Figure 4f). Whereas all particles adsorb to the interface of a bulk water-toluene mixture, the pronounced interfacial activity of particles with an increased wettability profile could be visually reflected in their increased driving force to expand the available surface area, which led to the particles "climbing up" the wall of the glass vials, creating an expanded area of amphiphilic Janus particles adsorbed at a thin layer of water on the hydrophilic glass in contact with the oil. In line with the $\zeta$-potential and contact angle measurements, these observations demonstrate the pronounced interfacial activity of the cup-shaped Janus particles with a high hydrophilic-to-hydrophobic surface ratio.

To further prove the nonuniform distribution of the chemical surface functionalities, we next exposed amphiphilic particles to a side-selective chemical postconjugation reaction scheme. Here, we opted for a bulk postfunctionalization reaction of carboxylic acid-presenting amphiphilic particles with a fluoresceinamine dye in an 1-ethyl-3-(3dimethylaminopropyl)carbodiimide (EDC) coupling reaction (Figure 5). Confocal micrographs of the functionalized particles revealed the anisotropic distribution of the fluorescent marker, selectively present at only the hydrophilic portions of the particle surface.

Both, the particle shape and surface functionality can impart colloidal interaction behavior. ${ }^{41,42}$ As such, we observed colloidal aggregation of Janus particles in both hydrophobic and hydrophilic environments. In hydrophobic solutions, the shape of the particles largely determined their organization. For instance, we observed that particles in the cup-shaped geometries packed into columnar stacks when dropped into toluene (Figure 6a). In turn, when Janus particles were mechanically forced into water, the colloidal aggregation behavior was dominated by hydrophobic interaction driven by self-assembly forces. As a result, in aqueous environments, marginal residues of oil sufficed to cause spontaneous selfassembly of amphiphilic Janus particles. Via targeted combination of different particles with variable shapes, this spontaneous colloidal self-assembly could be directed toward the predominant formation of dimeric, trimeric, or tetrameric particle assemblies, which could be readily observed in optical microscopy (Figures S8-S10). For instance, an immersion of hemispherical amphiphilic Janus particles resulted in a close to stochiometric formation of self-assembled dimers with the flat lipophilic interfaces of the particles facing each other (Figure 6b).

\section{CONCLUSIONS}

In summary, a straightforward one-step approach for the fabrication of chemically and physically anisotropic polymer particles is developed, wherein Janus emulsions act as particle molds allowing a fine-tuning of the shape, size, composition, and wettability profile of the final particles. The presented protocol of a phase-selective provision of functional monomers is general and broadly applicable, allowing the creation of a wide range of morphologically uniform nonspherical particles with a fine-tuned wettability profile. The fabrication procedure allows for various emulsification techniques and thus tailoring of particle sizes also at larger scale. All particles produced in this approach display an intrinsic Janus character, which enables side-selective postfunctionalization schemes, their directed assembly at fluid interfaces, and directional selfassembly into colloidal clusters. This broadened access to a wide range of amphiphilic Janus particles will help to rapidly prototype and deploy these colloidal materials in a variety of new and existing applications, for instance as potent Pickering emulsifiers, e.g., in oil recovery, for the engineering of structured surfaces and for directed self-organization of colloidal particles into functional meso- and macroscale assemblies.

\section{ASSOCIATED CONTENT}

\section{sI Supporting Information}

The Supporting Information is available free of charge at https://pubs.acs.org/doi/10.1021/acs.macromol.0c02152. 
Materials and methods, detailed experimental and characterization procedures, preparation of polymer substrates for contact angle measurements, postfunctionalization procedure, Pickering emulsion preparation, additional optical micrographs, and SEM images of amphiphilic Janus particles (PDF)

\section{AUTHOR INFORMATION}

\section{Corresponding Author}

Lukas Zeininger - Department of Colloid Chemistry, Max Planck Institute of Colloids \& Interfaces, 14476 Potsdam, Germany; (1) orcid.org/0000-0003-2339-5597;

Email: lukas.zeininger@mpikg.mpg.de

\section{Authors}

Bradley D. Frank - Department of Colloid Chemistry, Max Planck Institute of Colloids \& Interfaces, 14476 Potsdam, Germany

Markus Antonietti - Department of Colloid Chemistry, Max Planck Institute of Colloids \& Interfaces, 14476 Potsdam, Germany; 이이이.org/0000-0002-8395-7558

Complete contact information is available at: https://pubs.acs.org/10.1021/acs.macromol.0c02152

\section{Author Contributions}

The manuscript was written through contributions of all authors. All authors have given approval to the final version of the manuscript.

\section{Notes}

The authors declare no competing financial interest.

\section{ACKNOWLEDGMENTS}

The authors are thankful for funding by the Max Planck Society and gratefully acknowledge financial support through the Emmy-Noether program of the German Research Foundation (DFG) (grant no. ZE1121-3).

\section{REFERENCES}

(1) Walther, A.; Müller, A. H. E. Janus Particles: Synthesis, SelfAssembly, Physical Properties, and Applications. Chem. Rev. 2013, 113, 5194-5261.

(2) Wurm, F.; Kilbinger, A. F. M. Polymeric Janus Particles. Angew. Chem., Int. Ed. 2009, 48, 8412-8421.

(3) Binks, B. P.; Lumsdon, S. O. Influence of particle wettability on the type and stability of surfactant-free emulsions. Langmuir 2000, 16, $8622-8631$.

(4) Haney, B.; Werner, J. G.; Weitz, D. A.; Ramakrishnan, S. Absorbent-Adsorbates: Large Amphiphilic Janus Microgels as Droplet Stabilizers. ACS Appl. Mater. Interfaces 2020, 12, 3343933446.

(5) Tu, F.; Lee, D. Shape-Changing and Amphiphilicity-Reversing Janus Particles with $\mathrm{pH}$-Responsive Surfactant Properties. J. Am. Chem. Soc. 2014, 136, 9999-10006.

(6) Jeon, I.; et al. Janus Graphene: Scalable Self-Assembly and Solution-Phase Orthogonal Functionalization. Adv. Mater. 2019, 31, No. 1900438.

(7) Kumar, A.; Park, B. J.; Tu, F.; Lee, D. Amphiphilic Janus particles at fluid interfaces. Soft Matter 2013, 9, No. 6604.

(8) Howse, J. R.; Jones, R. A.; Ryan, A. J.; Gough, T.; Vafabakhsh, R.; Golestanian, R. Self-Motile Colloidal Particles: From Directed Propulsion to Random Walk. Phys. Rev. Lett. 2007, 99, No. 048102.

(9) Shields, C. W., IV; Velev, O. D. The Evolution of Active Particles: Toward Externally Powered Self-Propelling and SelfReconfiguring Particle Systems. Chem. 2017, 3, 539-559.
(10) Berger, S.; Synytska, A.; Ionov, L.; Eichhorn, K.; Stamm, M. Stimuli-Responsive Bicomponent Polymer Janus Particles by"Grafting from”/“Grafting to” Approaches. Macromolecules 2008, 41, 9669.

(11) Wang, P. Y.; Mason, T. G. Colloidal Lock-and-Key Dimerization Reactions of Hard Annular Sector Particles Controlled by Osmotic Pressure. J. Am. Chem. Soc. 2015, 137, 15308.

(12) Tang, C.; Zhang, C.; Sun, Y.; Liang, F.; Wang, Q.; Li, J.; Qu, X.; Yang, Z. Janus Anisotropic Hybrid Particles with Tunable Size from Patchy Composite Spheres. Macromolecules 2013, 46, 188-193.

(13) Nisisako, T.; Suzuki, H.; Hatsuzawa, T. Biconvex Polymer Microlenses with Tunable Imaging Properties Designed by Janus Droplet Microfluidics. Micromachines 2015, 6, 1435.

(14) Nagelberg, S.; et al. Reconfigurable and responsive dropletbased compound micro-lenses. Nat. Commun. 2017, 8, No. 14673.

(15) Zhang, Q.; Zeininger, L.; Sung, K. J.; Miller, E. A.; Yoshinaga, K.; Sikes, H. D.; Swager, T. M. Emulsion Agglutination Assay for the Detection of Protein-Protein Interactions: An Optical Sensor for Zika Virus. ACS Sens. 2019, 4, 180.

(16) Zeininger, L.; Weyandt, E.; Savagatrup, S.; Harvey, K. S.; Zhang, Q.; Zhao, Y.; Swager, T. M. Waveguide-based chemo- and biosensors: complex emulsions for the detection of caffeine and proteins. Lab Chip 2019, 19, 1327.

(17) Zeininger, L.; Nagelberg, S.; Harvey, K. S.; Savagatrup, S.; Herbert, M. B.; Yoshinaga, K.; Capobianco, J. A.; Kolle, M.; Swager, T. M. Rapid Detection of Salmonella enterica via Directional Emission from Carbohydrate-Functionalized Dynamic Double Emulsions. ACS Cent. Sci. 2019, 5, 789.

(18) Zhang, J.; Grzybowski, B. A.; Granick, S. Janus particle synthesis, assembly, and application. Langmuir 2017, 33, 6964.

(19) Hu, J.; Zhou, S.; Sun, Y.; Fang, X.; Wu, L. Fabrication, properties and applications of Janus particles. Chem. Soc. Rev. 2012, 41, 4356-4378.

(20) Gröschel, A. H.; Walther, A.; Lobling, T. I.; Schmelz, J.; Hanisch, A.; Schmalz, H.; Müller, A. H. Facile, Solution-Based Synthesis of Soft, Nanoscale Janus Particles with Tunable Janus Balance. J. Am. Chem. Soc. 2012, 134, 13850.

(21) Urban, M.; Freisinger, B.; Ghazy, O.; Staff, R.; Landfester, K.; Crespy, D.; Musyanovych, A. Polymer Janus Nanoparticles with Two Spatially Segregated Functionalizations. Macromolecules 2014, 47, 7194-7199.

(22) Ku, K. H.; Lee, Y. J.; Yi, G.-R.; Jang, S. G.; Schmidt, B. V. K. J.; Liao, K.; Klinger, D.; Hawker, C. J.; Kim, B. J. Shape-Tunable Biphasic Janus Particles as pH-Responsive Switchable Surfactants. Macromolecules 2017, 50, 9276-9285.

(23) Manoharan, V. N.; Elsesser, M. T.; Pine, D. J. Dense Packing and Symmetry in Small Clusters of Microspheres. Science 2003, 301, 483-487.

(24) Fan, J.-B.; et al. A general strategy to synthesize chemically and topologically anisotropic Janus particles. Sci. Adv. 2017, 3, No. e1603203.

(25) Song, Y.; Zhou, J.; Fan, J.-B.; Zhai, W.; Meng, J.; Wang, S. Hydrophilic/Oleophilic Magnetic Janus Particles for the Rapid and Efficient Oil-Water Separation. Adv. Funct. Mater. 2018, 28, No. 1802493

(26) Urbanski, M.; Lagerwall, J. P. F. Why organically functionalized nanoparticles increase the electrical conductivity of nematic liquid crystal dispersions. J. Mater. Chem. C 2016, 4, 3485-3491.

(27) Zheng, X.; Liu, M.; He, M.; Pine, D. J.; Weck, M. ShapeShifting Patchy Particles. Angew. Chem., Int. Ed. 2017, 56, 5507-5511.

(28) Kim, J.-W.; Larsen, R. J.; Weitz, D. A. Synthesis of nonspherical colloidal particles with anisotropic properties. J. Am. Chem. Soc. 2006, 128,14374

(29) Zhai, W.; Song, Y.; Gao, Z.; Fan, J.-B.; Wang, S. Precise Synthesis of Polymer Particles Spanning from Anisotropic Janus Particles to Heterogeneous Nanoporous Particles. Macromolecules 2019, 52, 3237-3243.

(30) Chern, C. S. Emulsion polymerization mechanisms and kinetics. Prog. Polym. Sci. 2006, 31, 443-486. 
(31) Yu, X.; Sun, Y.; Liang, F.; Jiang, B.; Yang, Z. Triblock Janus Particles by Seeded Emulsion Polymerization. Macromolecules 2019, 52, 96-102.

(32) Wang, X.; Bukusoglu, E.; Miller, D. S.; Pantoja, M. A. B.; Xiang, J.; Lavrentovich, O. D.; Abbott, N. L. Synthesis of Optically Complex, Porous, and Anisometric Polymeric Microparticles by Templating from Liquid Crystalline Droplets. Adv. Funct. Mater. 2016, 26, 73437351.

(33) Sun, Y.; Liang, F.; Qu, X.; Wang, Q.; Yang, Z. Robust Reactive Janus Composite Particles of Snowman Shape. Macromolecules 2015, $48,2715-2722$.

(34) Bradley, L. C.; Stebe, K. J.; Lee, D. Clickable Janus particles. J. Am. Chem. Soc. 2016, 138, 11437.

(35) Fan, J.-B.; Liu, H.; Song, Y.; Luo, Z.; Lu, Z.; Wang, S. Janus Particles Synthesis by Emulsion Interfacial Polymerization: Polystyrene as Seed or Beyond. Macromolecules 2018, 51, 1591-1597.

(36) Shum, H. C.; Abate, A. R.; Lee, D.; Studart, A. R.; Wang, B.; Chen, C.-H.; Thiele, J.; Shah, R. K.; Krummel, A.; Weitz, D. A. Droplet microfluidics for fabrication of non-spherical particles. Macromol. Rapid Commun. 2010, 31, 108.

(37) Dendukuri, D.; Doyle, P. S. The synthesis and assembly of polymeric microparticles using microfluidics. Adv. Mater. 2009, 21, 4071.

(38) Zarzar, L. D.; Sresht, V.; Sletten, E. M.; Kalow, J. A.; Blankschtein, D.; Swager, T. M. Dynamically reconfigurable complex emulsions via tunable interfacial tensions. Nature 2015, 518, 520.

(39) Wang, D.; Möhwald, H. Template-directed colloidal selfassembly-the route to 'top-down' nanochemical engineering. J. Mater. Chem. 2004, 14, 459.

(40) Sacanna, S.; Pine, D. J.; Yi, G.-R. Engineering shape: the novel geometries of colloidal self-assembly. Soft Matter 2013, 9, No. 8096.

(41) Wang, Y.; Wang, Y.; Breed, D. R.; Manoharan, V. N.; Feng, L.; Hollingsworth, A. D.; Weck, M.; Pine, D. J. Colloids with valence and specific directional bonding. Nature 2012, 491, 51.

(42) Wang, D.; Duan, H.; Möhwald, H. The water/oil interface: the emerging horizon for self-assembly of nanoparticles. Soft Matter 2005, 1, No. 412 . 